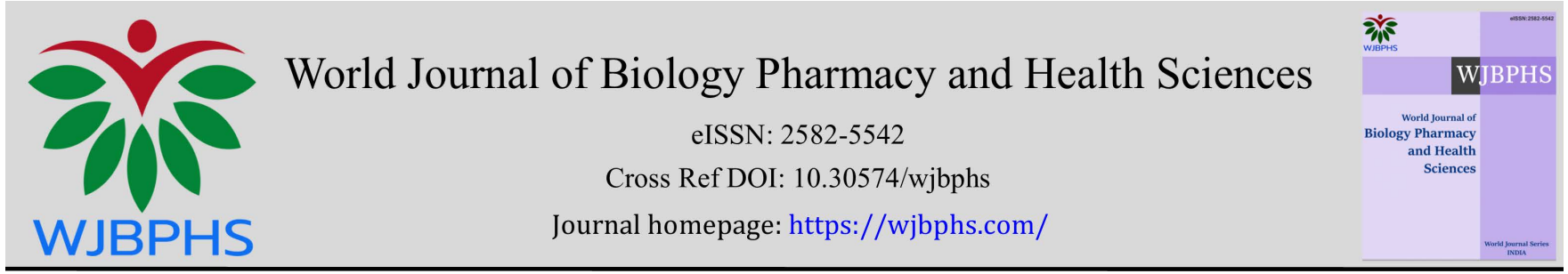

(RESEARCH ARTiCLE)

\title{
Prescription of the thyroid test results at the biochemistry laboratory in Antananarivo in 2018
}

\author{
Rakotoniaina TL 1, Ranaivosoa MK ${ }^{2,}{ }^{*}$, Rakotonindrina FI 3, Rakoto Alson OA 4 and Rasamindrakotroka A 5 \\ ${ }^{1}$ Medical Biology Student, Laboratory of Joseph Ravoahangy Andrianavalona University Hospital Antananarivo, \\ Madagascar. \\ ${ }^{2}$ Biologist, Laboratory of Biochemistry of Joseph Ravoahangy Andrianavalona University Hospital Antananarivo, \\ Madagascar. \\ ${ }^{3}$ Biologist, Laboratory Unit of University Hospital Center Mahavoky Atsimo Mahajanga, Madagascar. \\ ${ }^{4}$ Professor of Biological Haematology, Medical Biology Department of the Faculty of Medicine Antananarivo, \\ Madagascar. \\ 5 Professor of Immunology, Laboratory of Training and Research in Medical Biology, University of Antananarivo, \\ Madagascar.
}

World Journal of Biology Pharmacy and Health Sciences, 2021, 05(02), 019-024

Publication history: Received on 05 January 2021; revised on 04 February 2021; accepted on 06 February 2021

Article DOI: https://doi.org/10.30574/wjbphs.2021.5.2.0012

\begin{abstract}
According to National Authority for Health, the isolated dosage of TSH, in first-line, is a sufficient supply for the diagnosis and monitoring of thyroid dysfunction. The purpose of this study are to determine the prevalence of prescriptions of thyroid test, evaluate the practices on the prescription of thyroid tests compared to international recommendations. It is a descriptive retropective study within a period of 12 months. All the files with a request for TSH and / or thyroid hormone were included in this study. All files with a previous thyroid check-up or as part of a dysthyroidism follow-up assessment were excluded.
\end{abstract}

Among the 72600 prescriptions for biochemical tests, 184 corresponded to the prescription of thyroid tests, it means $0.25 \%$ compared to other biochemical blood tests recorded. Among the 184 prescriptions requesting thyroid tests, 117 files were retained. The mean age of the patients was 42.3 years, with a sex ratio of 0.18 .

One hundred sixteen files included a request of TSH dosage; 28,21\% included only a TSH dosage and 70.94\% included a request of simultaneous TSH dosage with one of two thyroid hormones. One prescription $(0.85 \%)$ asked for a thyroid hormones dosage only without preliminary TSH dosage.

TSH ranged from $<0.05$ to $93.97 \mu \mathrm{UI} / \mathrm{mL}$. It was normal in $68.96 \%$, reduced in $16.39 \%$ and increased in $14.65 \%$ of the dosages.

The number of thyroid hormone dosage in first-line in this study is important. Their prescription should be adapted to current recommendations in order to avoid the additional cost of unnecessary dosages for patients.

Keywords: Antananarivo; Biochemistry; Prescription; Thyroid function tests

\footnotetext{
* Corresponding author: Ranaivosoa MK

Biologist, Laboratory of Biochemistry of Joseph Ravoahangy Andrianavalona University Hospital Antananarivo, Madagascar. 


\section{Introduction}

The biological exploration of the thyroid function test essentially consists of TSH dosage wich can be completed by the dose of the thyroid hormones free fractions such as free thyroxine (FT4) and/or free triiodothyronine (FT3) [1]. The assay of these three hormones is carried out as part of the screening, diagnosis and monitoring of dysthyroidism (hyperthyroidism and hypothyroidism) [2]. According to National Authority for Health or HAS recommendation in 2014 [3-5] : the isolated dosage of TSH, in first-line, is a sufficient supply for the diagnosis and monitoring of thyroid dysfunction in almost all cases. The purpose of this study are to determine the prevalence of prescriptions of thyroid test, evaluate the practices on the prescription of thyroid tests compared to international recommendations.

\section{Materials and methods}

It is a descriptive retrospective study within a period of 12 months from January $1^{\text {st }}$

2018 to December 31st 2018, at the Paraclinical Training and Biochemistry Research Unit of The Joseph Ravoahangy Andrianavalona University Hospital Center (UPTR-JRA UHC). All the files with a request for TSH and / or thyroid hormone (FT4 and / or FT3) were included in this study. All files with a previous thyroid test or as part of a dysthyroidism follow-up assessment were excluded. The studied variables were the age, the gender, the clinical information and the results of the dosage of TSH, of FT3 and of FT4. We used the request files of the analysis, VIDAS ${ }^{\circledR}$ and the results records of TSH, of FT4 and FT3. The data were recorded on Excel Software, the data processing was carried on the SPSS software and Epi info 7.

For each patient, 4 to $5 \mathrm{~mL}$ of venous blood was sampled on a lithium heparin tube, forwarded to the laboratory in less than 2 hours, and then centrifuged at 3500prm for 10 minutes. The analysis of the plasma was done on VIDAS® using the ELFA technique (Enzyme Linked Fluorescent Assay). For TSH dosage, a third generation VIDAS®TSH kit was used with a detection limit of $0.05 \mu \mathrm{UI} / \mathrm{mL}$. The dosing principle combined a sandwich enzyme immunoassay method in one step to final fluoride detection. The reference values used by the laboratory were $9.0-20.0$ pmol/L for FT4, and 4.0 $8.3 \mathrm{pmol} / \mathrm{L}$ for FT3. All the values outside these limits were considered as pathological ones.

\section{Results}

\subsection{General outcomes}

During the study period, 72600 prescriptions for biochemical blood tests were received and 184 of them were thyroid tests (it means $0.25 \%$ )

\subsection{Socio-demographic characteristics of the study population}

The age of the patients ranged from 1 to 83 years old, the average age being 42.3 years old and there be rather a female predominance in our population study (sex - ratio 0.18 )

\subsection{Distribution of the patients according to the TSH value and the gender}

The reference values used by the laboratory were $0.25-5.00 \mu \mathrm{UI} / \mathrm{ml}$ for TSH.

TSH ranged from $<0.05$ to $93.97 \mu \mathrm{UI} / \mathrm{mL}$. It was normal in $68.96 \%$, reduced in $16.39 \%$ and increased in $14.65 \%$ of the dosages. 


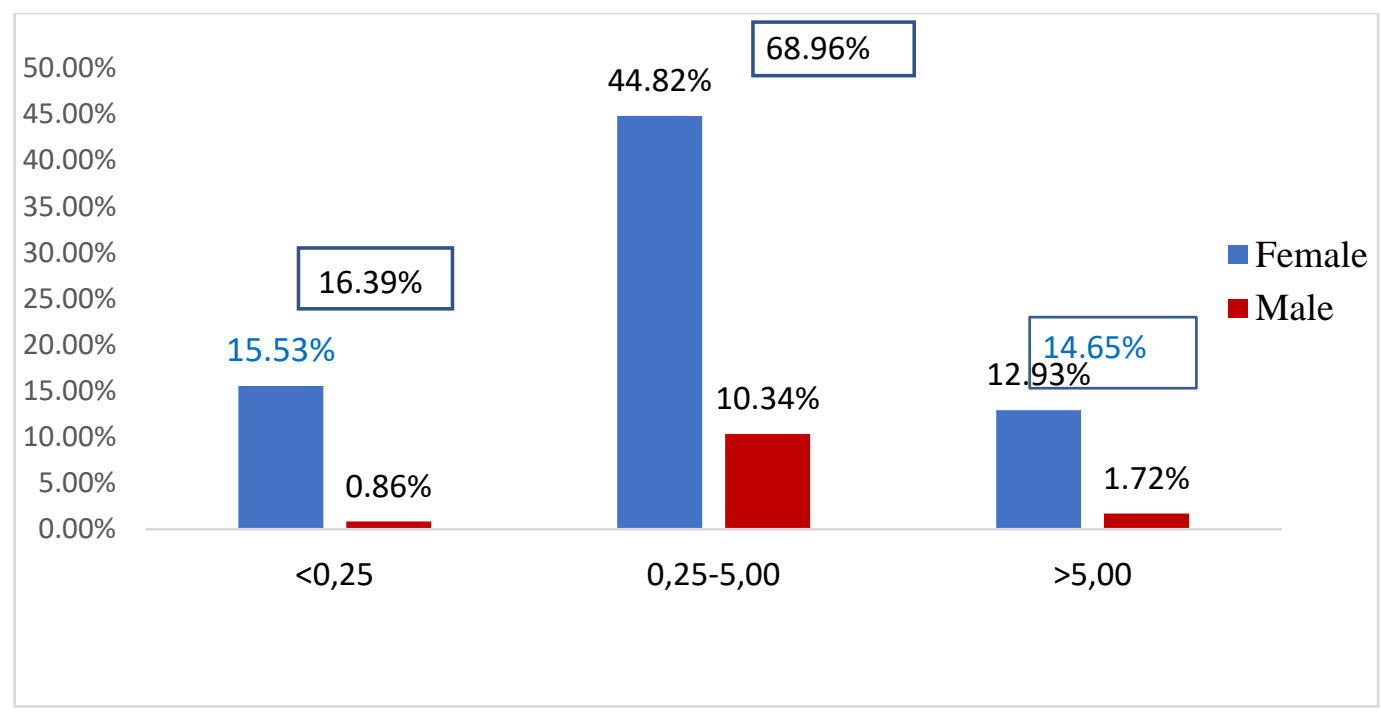

Figure 1 Distribution of the patients according to the TSH value and the gender

\subsection{Distribution of the patients according to the age and the TSH value}

A peak of TSH value> $5.00 \mu \mathrm{IU} / \mathrm{ml}$ was observed in the age between 40 to 69 years.

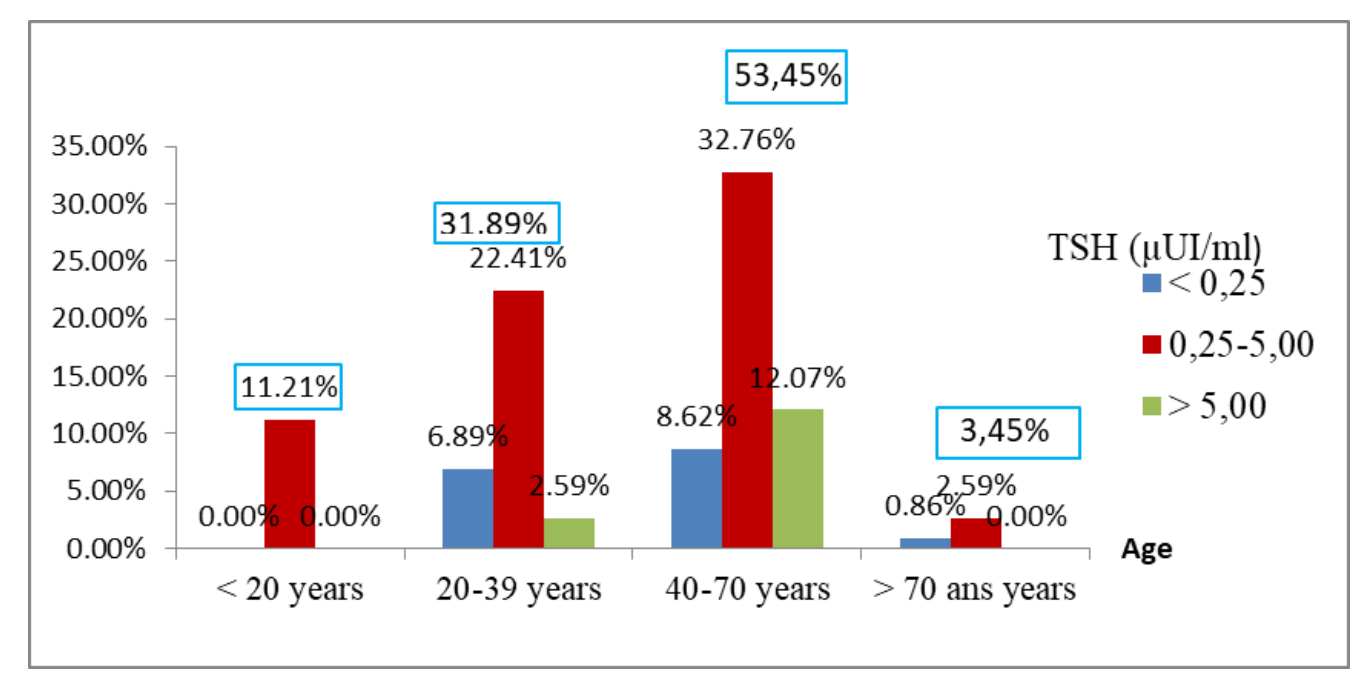

Figure 2 Distribution of the patients according to the age and the TSH value

\subsection{Prescription of the thyroid test}

One hundred sixteen files (it means $99.15 \%$ ) included a request of TSH dosage. $28.21 \%$ included only a TSH dosage and $70.94 \%$ included a request of simultaneous TSH dosage with one or two thyroid hormones. $35.89 \%$ were including TSH with FT4, 0\% with FT3 and 35.05\% of simultaneous dosage of the three hormones. One prescription (0.85\%) asked for a thyroid hormones dosage only without preliminary TSH dosage 
Table 1 Prescription of thyroid test

\begin{tabular}{|l|l|l|}
\hline Prescriptions & $\begin{array}{l}\text { Numbers } \\
(\mathbf{n = 1 1 7})\end{array}$ & $\begin{array}{l}\text { Percentage } \\
\mathbf{( 1 0 0 \% )}\end{array}$ \\
\hline TSH & 33 & $28.21 \%$ \\
\hline TSH + FT4 & 42 & $35.89 \%$ \\
\hline TSH + FT3 & 0 & $0.00 \%$ \\
\hline TSH +F T4 +FT3 & 41 & $35.05 \%$ \\
\hline FT4/FT3 & 1 & $0.85 \%$ \\
\hline
\end{tabular}

\subsection{Causes for prescriptions}

The reasons for prescriptions have been very diverse. Suspicion of hyperthyroidism was the first and most common reason for prescription, followed by suspicion of hypothyroidism.

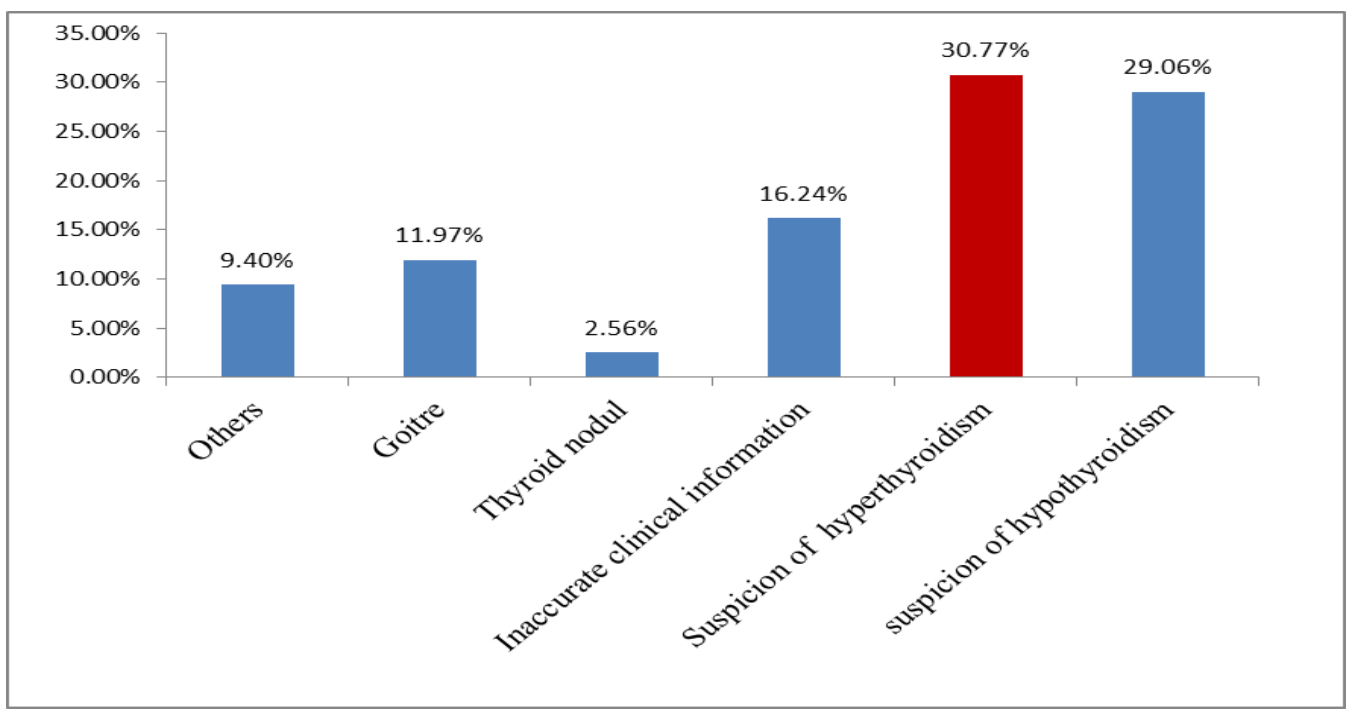

Figure 3 Distribution of prescriptions according to clinical information $(n=117)$

\subsection{Characteristic of the prescription according to TSH values}

TSH ranged from $<0.05$ to $93.97 \mu \mathrm{IU} / \mathrm{mL}$. It was normal in $68.96 \%$, it decreased in $16.39 \%$ and increased in $14.65 \%$ of the dosages.

Twenty-nine point ninety-one percent (35 cases) of dysthyroidism was revealed by the TSH dosage, $18.80 \%$ of which (22 cases) were found among prescriptions requiring simultaneous TSH dosage with one or both thyroid hormones. For the prescription without TSH dosage, thyroid hormones returned to normal $(0.85 \%)$.

\section{Discussion}

In the present study, 184 requests for thyroid examination out of 72600 biochemical prescriptions were found, corresponding to a prevalence of $0.25 \%$. In the United Kingdom, in 2015, a study conducted by Werhun A and HamiltonW found $6.37 \%$ of annual prescriptions for thyroid tests [6]. This low frequency of thyroid biology testing in our study compared to other biochemical tests could be linked to the high cost of thyroid testing, which costs around 9.22 to 10.47 euros. While in our country, the cost of care is borne by the patient's family, as in all developing countries where the health care system and social security are still non-existent, unlike in developed countries where social security is available to the entire population.

The pathological values of TSH in our study were mainly observed in female patients. These results are consistent with those found by Conffinier F et al. in Rouen in 2014 with a clear female predominance in these pathologies; 
hypothyroidism affects $5.1 \%$ of women and $0.92 \%$ of men, and $1.37 \%$ of women and $0.31 \%$ of men for hyperthyroidism [7]. Furthermore, the female gender is a risk factor for the development of dysthyroidism [8-10].

The most represented age group was between 50-59 years old. The average age was 42.3 years with extremes of 1 and 83 years. In the Fabris A. study conducted in France, the average age was 56 years with peaks of prescriptions in the age range between $40-49$ years and 60-69 years [8].

This study showed $28.21 \%$ of requests for thyroid tests containing a dosage of only TSH (that is 33/117) in accordance with the current recommendations. Other studies carried out in France, by Kasonga F. et al. and Fabris A. reported $87.49 \%(1000 / 1143)$ and $68.35 \%(108 / 158)$ respectively of only TSH requests $[11,8]$. Thus, in our study, $71.79 \%$ (or 84/117) of the thyroid tests did not follow the guidelines of good practice for the prescription of thyroid tests. This high frequency of prescribing thyroid tests that do not respect the recommended guidelines has an economic impact. Compliance with the recommendations on thyroid test prescription could have avoided the overcharge of dosages. In France in 2017, Kasonga $\mathrm{F}$ et al. identified $12.5 \%$ of prescriptions that did not respect the recommendations of the National Authority for Health. The non-compliance with the recommendations found in our study could be related to the lack of knowledge of the recommendations for thyroid test prescriptions.

Suspicion of hyperthyroidism was the primary reason for prescription in our case with $30.77 \%$ (36/117 prescriptions). A study conducted by Conffinier F et al. reported $21 \%$ suspicion of hyperthyroidism [7]. The main clinical signs of hyperthyroidism in our case were weight loss, malaise, hyperhydrosis, tachycardia, palpitations, heart rhythm disorder, anxiety, psychomotor agitation, behavioral disorder and neurotic state.

The suspicion of hypothyroidism was the second most common reason for prescription observed in our case with $29.06 \%$ of prescriptions (34/117). The main clinical signs leading to the suspicion of hypothyroidism in our series were isolated asthenia, weight gain, myalgia and constipation, miscarriages and pregnant vomiting. The clinical signs in favor of suspected hypothyroidism found in the literature were asthenia $(80.2 \%)$, weight gain $(48.1 \%)$, gastrointestinal disorders (31.3\%), muscle symptoms (24.9\%), and changes in skin status $(22.4 \%)[12,13]$.

Other authors have also reported asthenia as the predominant clinical sign in hypothyroidism suspicious patients although it is poorly sensitive and non-specific with low positive predictive value. [14-16].

Goitre was also a frequent reason for prescribing thyroid tests in our study with $11.97 \%$ (14/117). Among these requests, only one TSH value was decreased (TSH at $0.22 \mu \mathrm{UI} / \mathrm{ml}$ ).

Three checkups $(2.56 \%)$ were prescribed for thyroid nodule. None of these 3 tests met the recommendations for prescribing a thyroid test. Two of the 3 thyroid tests included TSH with T4L. The third one included TSH with T4L and T3L. In all cases; the TSH was always normal and did not require any additional requests according to the recommendations. No pathological values of TSH were observed, it was normal in all 3 cases.

In addition to the recommendations of the HAS, other authors point out that the first-line test to be requested in case of suspicion of dysthyroidism is the TSH and that its normality eliminates a priori a primitive dysthyroidism with a high negative predictive value [17]. Zulewski et al. have established and tested a clinical screening score for hypothyroidism but conclude that the TSH dosage is the best and most reliable test for detecting dysthyroidism [18]. Furthermore, the study by Meyerovitch et al. [19] shows that when the TSH level is normal, the probability that it becomes abnormal within 5 years is low $(2 \%)$.

\section{Conclusion}

In the present study, $71.79 \%$ (84/117) of thyroid biologic tests did not respect the guidelines of good practice. The respect of recommendations could have avoided the additional cost of dosages that were not essential for patients.

\section{Compliance with ethical standards}

\section{Acknowledgments}

We would like to thank all the staff of the laboratory of University Hospital of Ravoahangy Andrianavalona and all the laboratory technicians. Similarly, we would like to express our gratitude to the director of establishment for authorizing us to carry out this study. 


\section{Disclosure of conflict of interest}

All authors declare that they have no conflict of interest.

\section{References}

[1] Annaix V, Charpiot P. Biological exploration of the thyroid. Durand G, Beaudeux JL, eds. Medical biochemistry: current markers and perspectives. 2e édition. Paris: Lavoisier - Médecine Sciences Publications. 2011; 461-70.

[2] Herbomez M. Biological exploration of the thyroid. RevFrancoph Lab. 2009; 411: 39-44.

[3] National Authority for Health. Laboratory diagnosis and monitoring of hyperthyroidism in adults. HAS. 2000.

[4] National Authority for Health. Rough hypothyroidism in adults: diagnosis and management. HAS. 2007.

[5] National Agency for Accreditation and Health Evaluation. Laboratory diagnosis and monitoring of hyperthyroidism in adults. ANAES. Fév 2000.

[6] Werhun A, Hamilton W. Thyroid function testing in primary care: overused and under-evidenced? A study examining which clinical features correspond to an abnormal thyroid function result. Family practice. 2011; 32(2): 187-91.

[7] Coffinier F. Dysthyroidism in general practice: analysis of the study population ECOGEN [Thesis]. Médecine et pharmacie: Rouen. 2014.

[8] Fabris A. The prescription of thyroid tests in general medicine, Practice survey of 19 general practitioners in the Alpes-Maritimes [Thesis]. Médecine humaine: Nice. 2014.

[9] Estaquio C, Valeix P, Leenhardt L. Thyroid diseases in the SU.VI.MAX cohort: estimate of their impact, $1994-2002$. BEH. 2009; 41: 445-8.

[10] Goichot B. Prescribe and interpret the thyroid workup. Médecine Thérapeutique. 2016; 22: 259-64.

[11] Kasonga F, Cassinari K, Brunel V, François F, Frédéric Z. The correct prescription of the thyroid biological assessment as part of the diagnosis of dysthyroidism. Retrospective study at Rouen University Hospital. In Ann Biol Clin. 2018 July; 76(4): 421-8.

[12] Delemer B, Aubert JP, Nys P. An observational study of the initial management of hypothyroidism in France: the ORCHIDEE study. Eur J Endocrinol. 2012; 167(6): 817-23.

[13] Ladenson PW, Singer PA, Ain KB. American Thyroid Association guidelines for detection of thyroid dysfunction.Arch Intern Med. 2000; 160(11): 1573-5.

[14] Yazbeck R, Benoit P, Roche B. Primary hypothyroidism in adults over 60: Characteristics and follow-up after initiation of replacement therapy in a hospital setting. Press Med 1983. 2001; 30(24): 1193-7.

[15] Leclère J, Cousty C, Schlienger JL. Subclinical hypothyroidism and quality of life of women aged 50 or more with hypercholesterolemia: results of the HYOGA study. Press Med Paris Fr 1983. 2008; 37(11): 1538-46.

[16] Meyer P. Should subclinical dysthyroidism be treated?.Rev Med Suisse. 2008; 4: 14-7.

[17] Stockigt J. Assessment of Thyroid Function: Towards an Integrated Laboratory - Clinical Approach. ClinBiochemRev. 2003; 24(4): 109-22.

[18] Zulewski BM. Estimation of tissue hypothyroidism by a new clinical score: evaluation of patients with various grades of hypothyroidism and controls. J ClinEndocrinolMetab. 1997; 82(3): 771-6.

[19] Meyerovitch J, Rotman-Pikielny P, Sherf M. Serum thyrotropin measurements in the community: five-year followup in a large network of primary care physicians. Arch Intern Med. 2007; 167(14): 1533-8. 